\title{
Probing defects and correlations in the hydrogen-bond network of ab initio water
}

\author{
Piero Gasparotto, ${ }^{\dagger}$ \\ Ali A. Hassanali,,,+ and Michele Ceriotti ${ }^{*}, \dagger$ \\ Laboratory of Computational Science and Modeling, IMX, École Polytechnique Fédérale de \\ Lausanne, 1015 Lausanne, Switzerland, and The Abdus Salaam International Center for \\ Theoretical Physics, Condensed Matter Physics Section, Strada Costiera 11, Trieste Italy. \\ E-mail: ahassana@ictp.it; michele.ceriotti@epfl.ch
}

\begin{abstract}
The hydrogen-bond network of water is characterized by the presence of coordination defects relative to the ideal tetrahedral network of ice, whose fluctuations determine the static and time-dependent properties of the liquid. Because of topological constraints, such defects do not come alone, but are highly correlated coming in a plethora of different pairs. Here we discuss in detail such correlations in the case of ab initio water models and show that they have interesting similarities to regular and defective solid phases of water. Although defect correlations involve deviations from idealized tetrahedrality, they can still be regarded as weaker hydrogen bonds that retain a high degree of directionality. We also investigate how the structure and population of coordination defects is affected by approximations to the inter-atomic potential, finding that in most cases, the qualitative features of the hydrogen bond network are remarkably robust.
\end{abstract}

Keywords: ab initio, parallel tempering, hydrogen bond defects

\section{Introduction}

Many of the anomalous physical and chemical properties of water can be understood in terms of its highly-structured hydrogen-bond network. $\frac{112}{12}$ Tetrahedrally coordinated water, with two donated and two accepted H-bonds constitutes the fundamental building block of such networks. Of course, this idealized tetrahedral environment can be heavily distorted by thermal ${ }^{3]}$ and quantum ${ }^{4}$ fluctuations. In the liquid phase, coordination defects exist and their presence, concentration, and relative arrangement contribute to the structural and dynamical properties of water. ${ }^{21516}$ Here we investigate the properties of such coordination defects, with a particular focus on their structural correlations, by means of first-principles molecular dynamics simulations.

Over the last three decades, considerable effort and progress has been made in the simulation of liquid water from first principles calculations. In this regard, DFT-based ab initio simulations have elucidated the importance of several factors such as the quality of the electronic structure, the treatment of nuclear quantum effects (NQE) and also the role of statistical sampling ${ }^{7+-27}$ in reproducing the experimentally available oxygen-oxygen pair correlation function of water (RDF see Figure 11. Unlike the idealized tetrahedral structure of ice, finite temperature fluctuations create defects which are a small fraction of the $\mathrm{H}$-bond network and thus challenging to sample. Here we have taken exceptional precautions to ensure as extensive as possible thermodynamic sampling to collect meaningful statistics on the populations and 
structure of defects that in some cases contribute to less than a percent of the $\mathrm{H}$-bond network of liquid water. To achieve this, we used parallel tempering combined with the well-tempered ensemble $e^{28}$ (PTWTE) to perform an extensive sampling of a box of 64 and 128 water molecules.

Using different models of inter-atomic forces, or different thermodynamic conditions, may change slightly the typical geometry of a hydrogen bond. In order to make the structural definition of a hydrogen bond and the classification of the different coordination environments independent from these effects, we used an adaptive, probabilistic definition of the hydrogen bond (PAMM). ${ }^{29}$ Since in this work we restrict ourselves to a narrow range of thermodynamic conditions, this choice does not entail dramatic differences relative to one of the more traditional definitions, but provides a robust framework that would make it straightforward to perform a similar analysis for different systems or to investigate more dramatic changes in environmental factors. We also report on the impact of different details of the electronic structure calculation, although as we will see, for a given choice of exchange-correlation functional, the main factor contributing significantly to the structure and population of defects is the use or neglect of dispersion corrections. This is consistent with previous observations which have shown that the inclusion of dispersion corrections significantly reduces the over-structuring that is seen for the most common choices of exchange-correlation density functional 19]30|31

The topological constraints induced by the presence of an extended H-bond network mean that defects appear at highly correlated positions and are hence clustered together with different propensities. ${ }^{6[32}$ What is more, the details of the description of the inter-atomic forces do not significantly change the population of defects, nor their relative structural correlations. Although the RDF provides useful information on the structure of the system that can be readily compared with accurate experiments ${ }^{33}$ and is therefore regarded as the holy grail for benchmarking the quality of $a b$ initio models, it averages over all the underlying complexity of the topology of the HB network and its directional correlations. ${ }^{[34}$ As we will show here, one could consider the RDF as arising from the combination of correlations between different ideal or defective coordination environments. We present an extremely thorough analysis of such correlations, assessing the impact of many different computational details, and showing which of those matter, and which only cause small changes to the RDF but no profound qualitative change to the topological properties of the H-bond network. Furthermore, we use defect-resolved three dimensional distribution functions to elucidate the role of the weak interactions that are characteristic of under-coordinated environments. We find that the interactions between defects formed in the network have a remarkably directional character that could be interpreted as arising from 'weak' rather than altogether 'broken' hydrogen bonds - and link these angular correlations to those found in solid phases of water. While our analysis here focuses on thermodynamic, time-independent properties, we believe the structure and correlations of $\mathrm{H}$-bond defects will prove crucial to understand the fluctuations and hence dynamics of liquid water in future studies.

The paper is laid out as follows: we begin by describing the systems we simulated and the computational methods we used in Section 2. In Section 3.1 we catalogue the structural patterns in ab initio water obtained with the PAMM analysis. In Section 3.2 we use the patterns detected by PAMM to infer structural correlations between defects in water's hydrogen bond network. In Section 4 we compare the distribution of coordination defects produced by different water models varying the level of theory used for the electronic structure, finite box size and nuclear quantum effects. Finally we end with some concluding remarks on our work in Section 5 .

\section{Computational Methods}

In this work we have used AIMD simulations based on density functional theory (DFT) coupled with PTWTE. We will begin by first summarizing details of the electronic structure methods used and then describe the protocol we applied for the PTWTE simulations. 


\subsection{Ab Initio methods}

The electronic structure calculations for computing the energies and forces were conducted using Quickstep which is part of the CP2K package. ${ }^{35}$ The molecular dynamics and parallel tempering simulations were performed using the recently released code i-PI, that decouples the calculation of the interatomic forces from the dynamic evolution of the nuclei $\cdot \frac{36}{} \mathrm{~A}$ convergence criterion of $5 \times 10^{-7}$ a.u was used for the optimization of the wavefunction in all the simulations. Unless otherwise stated, the wavefunction was expanded in a DZVP Gaussian basis set, and an auxiliary basis set of plane waves was used to expand the electron density up to a cutoff of 300Ry. The D3 Grimme dispersion corrections ${ }^{37}$ for the van der Waals interactions were used for most of the simulations. We used the BLYP generalized gradient correction ${ }^{38}$ to the local density approximation and Goedecker-Teter-Hutter (GTH) pseudopotentials. ${ }^{39}$ All simulations were thermostated within the NVT ensemble using the canonical-sampling velocity-rescaling thermostat ${ }^{40]}$ with a time constant of $1 \mathrm{fs}$. To maximize sampling of uncorrelated potential energy structures and accelerate replica exchanges in PT simulations, we also included a generalized Langevin equation thermostat tuned for efficient sampling. $\frac{41.42}{4}$

Extensive tests of the sensitivity of results to different computational details were performed using a box of side length $12.4138 \AA$ with 64 water molecules, corresponding to the experimental density of the system at $300 \mathrm{~K}$. The different PT simulations that were conducted included the following: BLYP without D3 Grimme's dispersion correction (BLYP+NOVDW), BLYP with dispersion corrections (BLYP+VDW), BLYP+VDW simulations with the TZV2P basis set (BLYP+VDW+TZV2P) and finally BLYP+VDW simulations using a plane wave cutoff of 350Ry (BLYP+VDW+350). For all the previously described PT runs, a timestep of $1 \mathrm{fs}$ was used. Most AIMD simulations using Born Oppenheimer molecular dynamics use a smaller timestep of $0.5 \mathrm{fs}$, which is necessary to obtain accurate real-time dynamics, but does not change significantly structural properties. In order to assess the sensitivity of our results to the choice of a larger timestep than commonly used, PT simulations were also conducted using BLYP+VDW with a timestep of $0.5 \mathrm{fs}$ (BLYP+VDW+0.5fs) which shows that using a larger timestep does not qualitatively change the structural properties of the system such as the diversity of different defects in the system (for more details see Figure. S3 of the SI).

The PT runs for the 64 water boxes detailed above were used to identify parameters for our production simulations with a larger box. We thus also performed parallel tempering simulations of 128 water molecules with a box size of $15.6404 \AA$ using BLYP+VDW, DZVP, 300Ry cutoff and 1fs time step. This simulation will be referred to as PTL. From our PTL simulations, we initiated four independent PIGLET simulations, using six beads and colored-noise thermostatting, $\frac{43}{4}$ to assess the role of nuclear quantum effects (NQE).

Besides the simulations using the BLYP functional, we also conducted simulations with the more expensive hybrid functional B3LYP implemented in $\mathrm{CP} 2 \mathrm{~K},{ }^{44}$ with $\mathrm{D} 3 \mathrm{vdW}$ corrections, in order to ascertain the role of electron exchange on the properties of liquid water. Due to the high cost of including Hartree-Fock exchange, we could not run replica-exchange simulations. Instead, we ran four independent simulations of about 16ps each, starting from BLYP equilibrated configurations extracted from the BLYP+VDW PT simulation consisting of 64 waters, and discarding the first 2 ps for equilibration with the hybrid functional.

Although the combination of hybrid functionals and dispersion corrections appear to improve the properties of ab initio water, we wanted to ascertain whether the defect correlations were not exclusive to DFT simulations. Hence, to complement our AIMD simulations, we also investigated populations and correlations of defects using force field models. Namely, we performed a classical simulation of 512 water molecules using a fixed point-charge model (TIP4P/2005 $\mathrm{f}^{45}$ ) and classical and PIGLET simulations of 216 water molecules using MB-pol, a sophisticated force-field based on a many-body expansion and high-end quantum chemical reference calculations. $46-48$ For nonreactive simulations of water, $\mathrm{MB}-\mathrm{Pol}$ is probably the most realistic theoretical model of the be- 
haviour of molecular $\mathrm{H}_{2} \mathrm{O}$. Finally, we also make some comparisons of the topological defects found in liquid water to the coordination environment found in solid phases of ice.

In what follows, we will focus mostly on the results of the PTL simulations with 128 waters and make reference to the analysis of the temperature dependence and electronic structure in the text, but deferring most of the details to the SI. Other simulation details, including the lengths of all the simulations conducted, are summarized in Table I of the SI.

\subsection{Parallel tempering protocol}

$\mathrm{Ab}$ initio parallel tempering simulations for the 64 water boxes were performed using six replicas at the following temperatures: 290, 304, 322, 343, $365,390 \mathrm{~K}$. In parallel tempering, a series of replicas are simulated at these six temperatures and thereafter exchanges between adjacent replicas are performed using a Metropolis criterion. In order to achieve efficient exchange between the replicas there must be significant overlap in the potential energy distributions of neighboring temperatures. Since a larger system exhibits smaller (relative) energy fluctuations, for PTL we used 8 replicas at the temperatures 290, 300, 310, 322, 335, $351,369,390 \mathrm{~K}$. It has recently been shown that by combining parallel tempering with the well tempered ensemble (PT+WTE), the overlap in energy between adjacent replicas is increased resulting in more frequent exchanges. $\frac{28}{28}$ In the well tempered ensemble the enhancement of the fluctuations in energy is tuned by the $\gamma$ factor which in our case was chosen to be 4 . Exchanges between the replicas were attempted every MD step.

AIMD simulations are still prohibitively computationally expensive and hence it is rather challenging to perform a systematic benchmarking of parameters to tune the optimal number of replicas used as well as the $\gamma$ factor. We decided to adopt a simplified protocol to determine the bias to generate the well-tempered ensemble. The mean and fluctuations of potential energy as a function of temperature were determined based on a short (5ps) preliminary run. Then, a fixed bias was constructed and applied to the remainder of the simulation. Rather than using a (divergent) parabolic bias, we decided to give it a Gaussian shape with mean and curvature compatible with the measured fluctuations, but designed to cut off to zero for large fluctuations. Specifically, we used

$$
B(V)=k_{B} T(\gamma-1) \exp \left[-\frac{1}{2 \gamma \delta V^{2}}(V-\bar{V})^{2}\right],
$$

where $\bar{V}$ and $\delta V^{2}$ are respectively the mean and variance of the potential energy evaluated for a given parallel tempering replica.

\section{Structural patterns in ab ini- tio water}

Let us start by introducing the different types of pair correlation functions that will be discussed in the rest of the paper. The left-most panel of Fig. 1 illustrates the familiar oxygen-oxygen pair RDF obtained from the PTL simulations at $300 \mathrm{~K}$. The middle panel of Fig. 1 shows instead the 3D oxygen-oxygen correlation function, which contains information on angular correlations that are lost in the RDF. The contour plot in the right-most panel shows a cut of such a 3D correlation function in the plane of the molecule (xy) and along the orthogonal symmetry plane $(x z)$. The middle and right panels quantify the orientation of water molecules either accepting or donating hydrogen bonds in the first hydration shell of a particular water. The peaks at positive $x$ correspond to the position of water molecules to which the tagged water is donating a hydrogen bond, and the broader peaks at negative $x$ values involve the accepting side of the central molecule. This is consistent with the notion of a ring of delocalized electron density, refered to as the 'negativity track' created by the lone-pairs which permits a larger range of local tetrahedrality. ${ }^{[2}$ Note that a peak in the density from both the donating and accepting side is a signature of highly directed nature of the hydrogen bonds. The importance of highlighting this feature will become clear when we show similar distributions for clustered defects.

One of the crucial observations we make is that there are significant correlations amongst defects in the hydrogen bond network. Since these defects tend to be rare and fleetingly lived and char- 

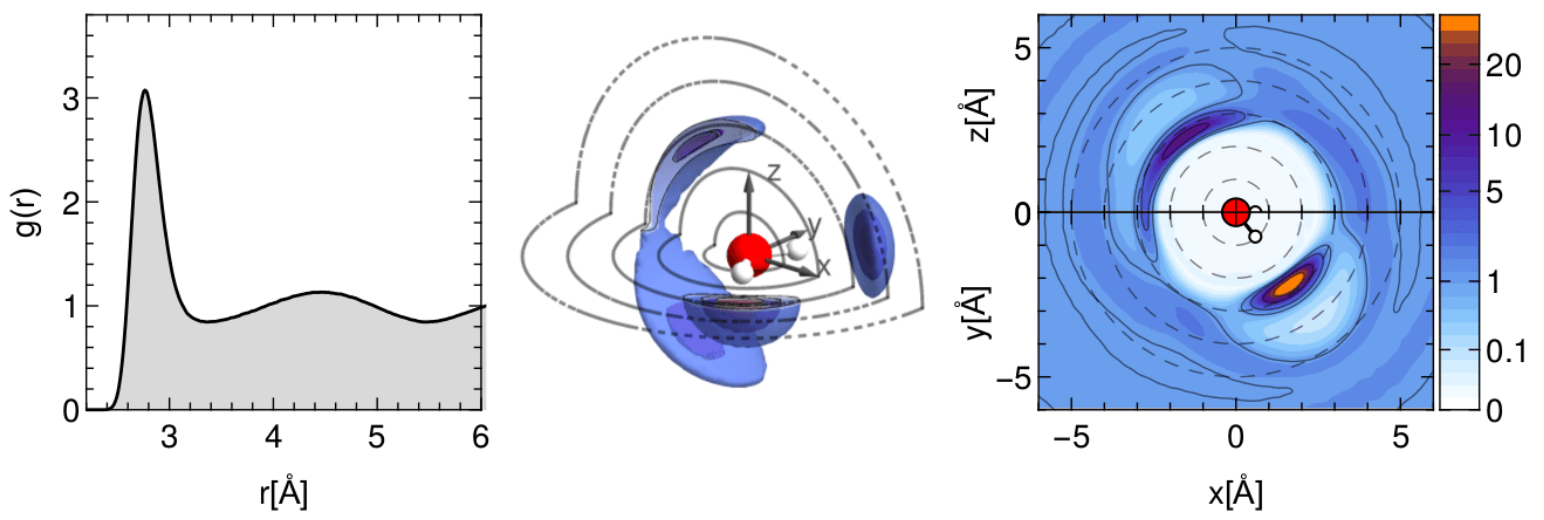

Figure 1: Oxygen-oxygen radial distribution functions computed at $300 \mathrm{~K}$ from PTL (BLYP+VDW+PTWTE-128) runs. Left to right, the figures correspond to the usual 1D radial distribution, to the 3D distribution and finally to slices of the 3D distribution along the $x y$ and $x z$ molecular planes. Dashed circles are a drawn as a guide for the eye, indicating a radial grid with a $1 \AA$ spacing.

acterized by geometrical properties that are possibly different from idealized tetrahedral water, we wanted to ascertain how structurally intact or well-defined hydrogen bonds change under different definitions or thermodynamic conditions. In a recent study, it was proposed that an unbiased definition of general fingerprints functions for recurring molecular patterns could be obtained automatically from an analysis of simulation data. Such a probabilistic analysis of molecular motifs $(\mathrm{PAMM})^{29}$ was shown to give an adaptive definition of $\mathrm{H}$ bonds, that could be made fully consistent with systems as diverse as alanine dipeptide, classical and quantum water, and liquid ammonia. The reader is referred to the original paper for details of the method, which is based on the automatic identification of local maxima in the probability distribution in pattern configuration space, followed by optimization of a Gaussian mixture model. The posterior probabilities give a natural partitioning of the configuration space over stable, recurring molecular patterns.

This definition has many advantages over more traditional ones. Firstly, it is probabilistic in nature and fuzzy: each $\mathrm{O}-\mathrm{H} \cdots \mathrm{O}^{\prime}$ triplet is assigned a fingerprint that varies smoothly between 0 (no HB) and 1 (clear-cut HB). Secondly, it is adaptive: since it detects modes of the probability distribution in configuration space, the definition will change depending on temperature and water model, separating clearly the slight model dependence of HB geometry and the changes in popula- tions of defects. Whereas a conventional geometrical definition would require a manual adjustment of its parameters, $\frac{49}{4}$ the PAMM algorithm determines automatically, for each simulation scenario, which range of geometries should be considered as a hydrogen bond. For the simulations conducted in this specific study - which is performed at the thermodynamic conditions to which the traditional hydrogen-bond definitions have been tuned - the choice of a PAMM definition over a conventional bond-angle definition introduces only minor differences and does not change our conclusions (see Section. 3 in SI).

\subsection{Population of coordination defects}

The PAMM methodology provides a convenient approach to define three main types of $\mathrm{H}$-bond count functions, $s_{\mathrm{D}}, s_{\mathrm{A}}$, and $s_{\mathrm{H}}$. $s_{\mathrm{D}}$ quantifies the total number of HBs donated by a tagged $\mathrm{O}$ atom, $s_{\mathrm{A}}$ the number of HBs accepted by an atom and finally $s_{\mathrm{H}}$ quantifies the number of HBs that any particular hydrogen participates in. 29 These are obtained by summing the value of the PAMM HB fingerprint computed for all possible donor$\mathrm{H}$-acceptor triplets involving the tagged atom ${ }^{1}$ Based on these counts, one can build very informative defect stability maps as seen in Fig. 2, that

\footnotetext{
${ }^{1}$ Of course, in practical implementations, a cutoff and a neighbor list can be used to maintain a linear scaling computational complexity of the analysis.
} 

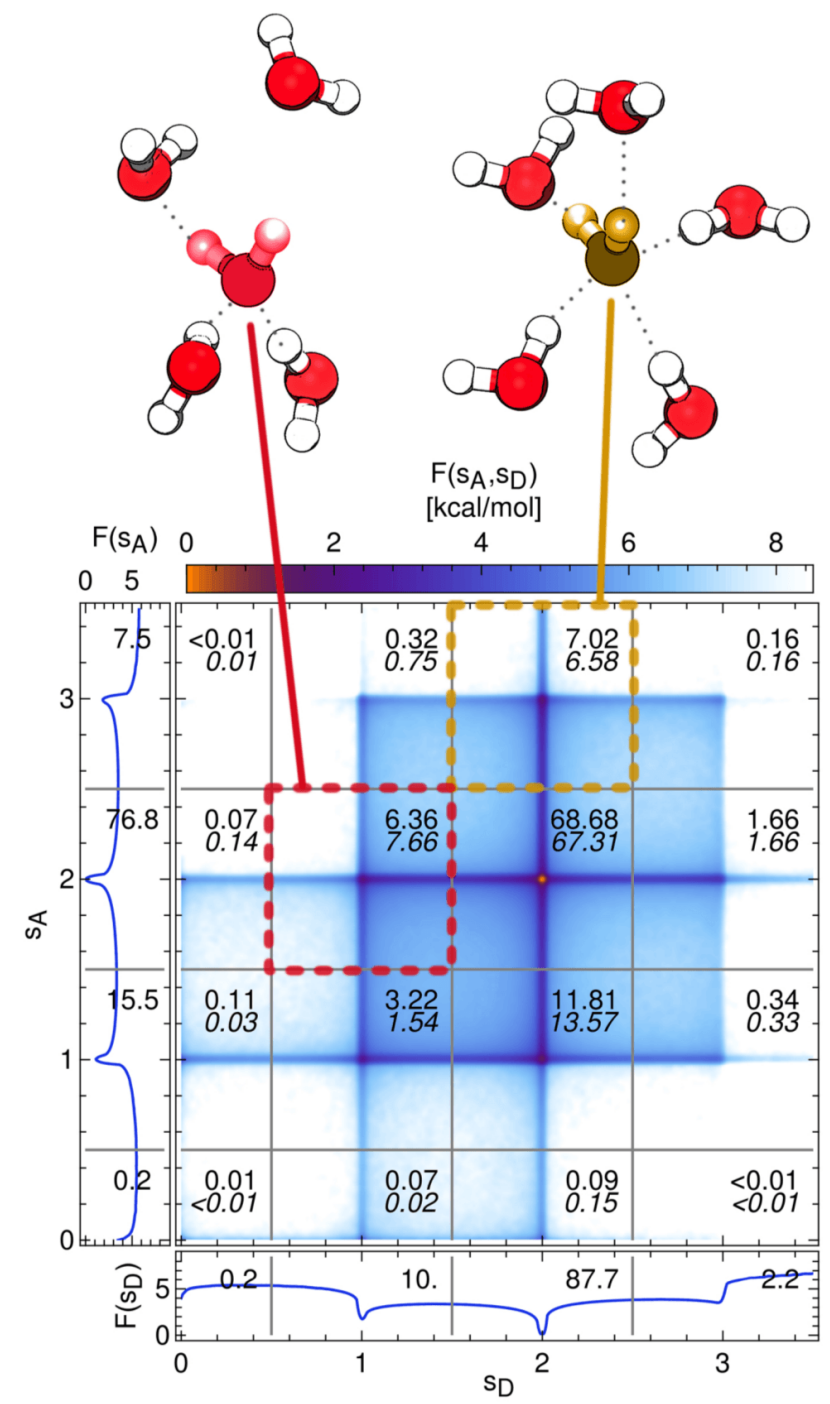

Figure 2: H-bond coordination summary for the PTL simulation data at $T=300 \mathrm{~K}$. $s_{\mathrm{D}}$ counts the $\mathrm{H}$-bonds donated by a $\mathrm{O}$ atom, and $s_{\mathrm{A}}$ those accepted. Fractional values characterize fluctuations. The $\left(s_{\mathrm{D}}, s_{\mathrm{A}}\right)$ range is partitioned in discrete regions that are assigned to different coordination states. For instance, the region with $0.5 \leq s_{\mathrm{D}}<1.5$ and $1.5 \leq s_{\mathrm{A}}<2.5$ is assigned to the $1_{\mathrm{D}} 2_{\mathrm{A}}$ state. The numbers reported in each region correspond to the overall fraction of $\mathrm{O}$ atoms observed in a given state (in percent), while the number in italics is the corresponding value obtained as a product of the marginal probabilities. The larger the difference between the two values, the larger the correlations that exist between the donor and acceptor counts, for a given coordination state. summarizes the relative probabilities of finding a water molecule in one of different coordination states. It is clear that for the PTL simulations at $300 \mathrm{~K}$, most water molecules donate and accept 2 hydrogen bonds. However, there is a sizable fraction of different types of topological defects. A nice feature of this type of analysis is that one can immediately point to asymmetries in the accepting vs donating abilities of hydrogen bonds - for example, there is a higher probability of finding water molecules that accept 2 and donate 1 hydrogen bond compared to those that accept 1 and donate 2 hydrogen bonds. We also see clearly the asymmetry in the distributions associated with a water molecule being a donor or acceptor which is consistent with previous observations by Agmon. ${ }^{2}$ Similar maps for other simulations at a higher temperature and electronic structure approximations are shown in the SI.

An important advantage of PAMM relative to traditional $\mathrm{H}$-bond definitions is that since the underlying fingerprint is probabilistic, and varies smoothly between zero and one, it is possible to recognize features in the transition regions between clear-cut defect states. The smooth definition of PAMM provides a qualitative description of the pathway from one defect state to another. Even though an order parameter based on a single site cannot capture quantitatively the complex collective modes that underlie the rearrangement of the H-bond network, $\underline{50}$ the height of the barrier between two defect states gives an indication of the relative propensity towards a transition. For instance, one can see that in the overwhelming majority of cases the state of an $\mathrm{O}$ atom evolves by increasing or decreasing by one the donated or accepted hydrogen-bond count, while concerted transitions do not contribute significantly. By looking at cuts in the free energy surface at constant $s_{\mathrm{A}}$ or $s_{\mathrm{D}}$, one can also get an idea of how the free energy barriers along the pathways to make or break hydrogen bonds change for molecules that are initially undercoordinated or overcoordinated (see Figure S1 in the SI). For instance, the barrier for the $1_{\mathrm{D}} 2_{\mathrm{A}} \rightarrow 1_{\mathrm{D}} 1_{\mathrm{A}}$ transition is lower by $\sim$ $33 \%$ than the barrier for the $2_{\mathrm{D}} 2_{\mathrm{A}} \rightarrow 2_{\mathrm{D}} 1_{\mathrm{A}}$ transition. Similarly, the barrier for the $2_{\mathrm{D}} 1_{\mathrm{A}} \rightarrow 1_{\mathrm{D}} 1_{\mathrm{A}}$ transition is lower than the $2_{\mathrm{D}} 3_{\mathrm{A}} \rightarrow 1_{\mathrm{D}} 3_{\mathrm{A}}$ transition roughly by a factor of two. Interestingly, these 
qualitative trends appear to be quite robust to the choice of different approximations made in treating the underlying electronic potential. However, it should be noted that small differences in these barriers will lead to much larger changes in dynamical properties, thus making it even more challenging to achieve statistical convergence.

Although we will not exploit this aspect here, it is worth stressing that strictly speaking, PAMM identifies coordination of $\mathrm{O}$ atoms, rather than water molecules, which means that this classification could be used transparently also in the presence of charged defects and charge fluctuations, since one is not relying on the definition of molecular entities. For instance, in the presence of an excess proton, one would expect to see an increase in density in the region with $s_{\mathrm{D}} \approx 3$ and $s_{\mathrm{A}}<1$, or detect the quantum fluctuations of a proton along a hydrogen bond by the appearance of diagonal features (see Ref. ${ }^{29}$ ) that correspond to one hydrogen bond momentarily changing its character from acceptor to donor.

\subsection{Defect correlations and the RDF}

The hydrogen bond maps of $s_{A}$ and $s_{D}$ shown in Fig. 2 provide a convenient way to classify water environments based on their coordination state. $\frac{5152}{152}$ Given that the probability maxima are very clear-cut and with a rather obvious structure as seen in Fig. 2, we subdivided the map manually labelling e.g. $n_{\mathrm{D}} m_{\mathrm{A}}$ an oxygen atom that has $s_{\mathrm{A}} \in\left[m_{\mathrm{A}}-0.5, m_{\mathrm{A}}+0.5\right)$ and $s_{\mathrm{D}} \in\left[n_{\mathrm{D}}-0.5, n_{\mathrm{D}}+0.5\right)$.

Armed with this classification, we can proceed to investigate whether different $n_{\mathrm{D}} m_{\mathrm{A}}$ states are distributed randomly in the network, or whether significant correlations exist between them. Twobody spatial correlation functions provide powerful tools to recognize such correlations, and allow us to disentangle the underlying factors that control the shape of the overall O-O RDF shown in Fig. 1 .

The simplest analysis involves coordinationresolved RDFs $n_{\mathrm{D}} m_{\mathrm{A}}-n^{\prime}{ }_{\mathrm{D}} m_{\mathrm{A}}^{\prime}-$ that report on the probability of finding an $\mathrm{O}$ atom in a coordination state $n_{\mathrm{D}} m_{\mathrm{A}}$ and another in $n_{\mathrm{D}}^{\prime} m_{\mathrm{A}}^{\prime}$ at a distance $r$ from one another. In addition, one can also look at the 3D distributions, as shown in Fig. 1. which give deeper insight into the angular position of waters within the first hydration shell. We will label as $n_{\mathrm{D}} m_{\mathrm{A}} \rightarrow n^{\prime}{ }_{\mathrm{D}} m^{\prime}{ }_{\mathrm{A}}$ the distribution of $n_{\mathrm{D}}^{\prime} m_{\mathrm{A}}^{\prime}$ evaluated in the reference frame of a $n_{\mathrm{D}} m_{\mathrm{A}}$ molecule. It is important to recognize that for each pair of species, the distributions associated with $n_{\mathrm{D}} m_{\mathrm{A}}$ and $n_{\mathrm{D}}^{\prime} m_{\mathrm{A}}^{\prime}$ are $n o t$ symmetric. If there is enhanced probability of finding $n_{\mathrm{D}}^{\prime} m_{\mathrm{A}}^{\prime}$ waters in the donor region of a $n_{\mathrm{D}} m_{\mathrm{A}}$ molecule, one can expect that viewed from the point of view of $n_{\mathrm{D}}^{\prime} m_{\mathrm{A}}^{\prime}$ this same correlation will amount to an enhancement in the acceptor region. Figure 3 provides an example of this kind of analysis, where we consider correlations between an un-specified $\star_{D} \star_{A} O$ atom and the main coordination states. The complete series of radial and 3D correlation functions, for all temperatures and electronic structure methods we considered in the present study, are provided in the SI. Here we will only comment on the most significant correlations, that can shed some light on the complex topological features of the $\mathrm{H}$ bond network of liquid water.

Let us start by commenting on the $\mathrm{O}-\mathrm{O}$ radial distribution functions. It is tempting to analyze the changes of the overall O-O RDF with temperature and simulation details in terms of the components resolved into the different coordination states. Contrary to the inherent structure analysis, that identifies "low-density/high-tetrahedrality" and "high-density/low-tetrahedrality" by quenching instantaneous liquid configurations, $\frac{53}{6}$ the analysis we perform here includes snapshots that are fully consistent with the given thermodynamic state point. We have seen in Section 3.1 that the majority of water molecules corresponds to thermal fluctuations around a tetrahedral $2{ }_{\mathrm{D}} 2_{\mathrm{A}}$ environment. All defective environments conspire to modify the shape of the short-range region of the RDF: over-coordinated defects do so by broadening the first peak, but would themselves increase the depth of the first minimum. Undercoordinated environments, instead, enhance the density in the interstitial region. This analysis thus provides an alternative interpretation of the structure of the RDF in terms of correlations between coordination defects on top of a dominant tetrahedral network, without invoking explicitly the existence of two thermodynamically distinct low and highdensity water networks. As can be seen in the SI, 


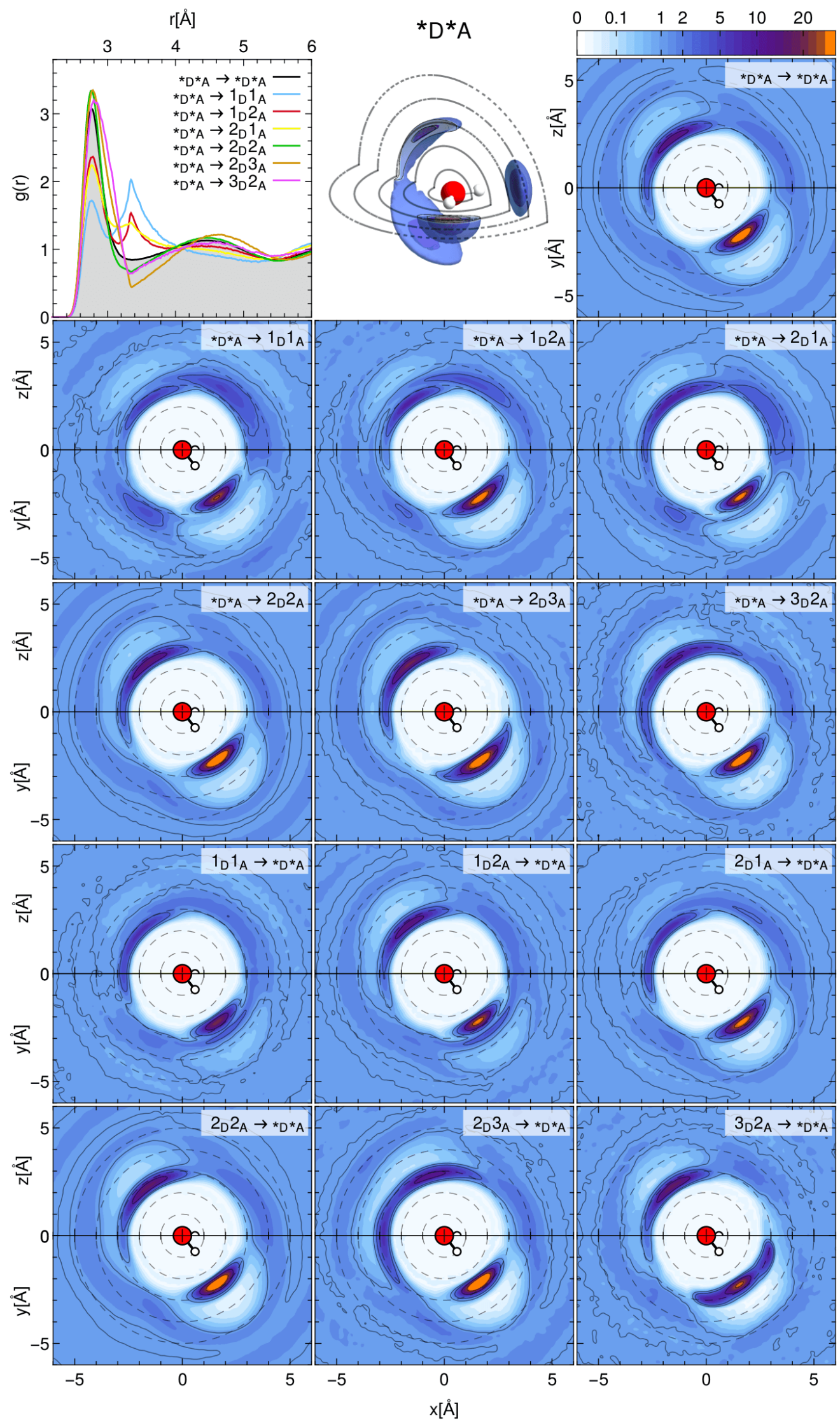

Figure 3: The figure reports concisely the $\mathrm{O}$ $\mathrm{O}$ correlation functions involving a defective $\mathrm{H}$-bond $\mathrm{O}$ environment, and a second oxygen atom without specification of its H-bonding state. The first row reports the baseline $\mathrm{O}$ $\mathrm{O}$ correlations, as in Fig,1. The 1D radial distribution function reports all the distributions of oxygen atoms around the most important defect environments, with the baseline shaded in grey. The following six panels report slices along high-symmetry molecular planes of the 3D distribution of the various defects around a non-specified $\mathrm{O}$ atom, while the last six report the mirror distribution of an arbitrary $\mathrm{O}$ atom around the specified defective species. 
changing the simulation temperature, or the details of the electronic structure, modulate both the populations and the shape of the RDF of defect resolved individual components, although many of the qualitative features associated with the relative population of defects as well as the strutural correlations between them, are still conserved (see SI for details).

It is perhaps worth stressing that any decomposition of this kind that dissects a structural observable based on prior structural analysis, risks being tautological to some extent. For instance, the lowering of the first peak, and appearance of a second peak in the interstitial region for undercoordinated defects could be regarded just as hydrogen bonds caught in the act of breaking up, rather than as a significant feature in the water network. An analysis of the 3D DF can identify more clearly the nature of the correlations between defects, telling apart artifacts of the analysis from genuine features of the H-bond network.

Focusing first on the under-coordinated water environments, and looking in the direction of the donated $\mathrm{H}$-bond in the $1_{\mathrm{D}} 2_{\mathrm{A}} \rightarrow{ }{ }_{\mathrm{D}} \star_{\mathrm{A}}$ and $1_{\mathrm{D}} 1_{\mathrm{A}} \rightarrow$ ${ }^{\star} \mathrm{D}{ }^{\star} \mathrm{A}$ correlation functions, one can see a sharp second peak that could indeed be interpreted as arising from the tail of the distribution of a "normal" H-bond, that is identified as broken by the PAMM fingerprint function (or the conventional structural definition). On the other hand, inspection of the mirror distributions $\star_{\mathrm{D}^{\star}}{ }_{\mathrm{A}} \rightarrow 1_{\mathrm{D}} 2_{\mathrm{A}}$ and $\star_{\mathrm{D}} \star_{\mathrm{A}} \rightarrow 1_{\mathrm{D}} 1_{\mathrm{A}}$ does not show a similar sharp peak just next to the "normal" acceptor peak. Rather, it reveals the presence of strong angular correlations in anomalous directions, that could be regarded as the manifestation of a weaker type of $\mathrm{H}$-bond rather than truly unbound configurations or broken hydrogen bonds. Even though all simulations in the present work were thermostatted, and so it is not possible to extract rigorous dynamical information, it is clear from inspection of the trajectories that these weak hydrogen bonds, while forming a smaller part of the population in the hydrogen bond network, are not just fleetingly formed transition-state structures, but rather involve metastable states. It would be interesting to perform an energy decomposition analysis as has been done in previous studies, $\frac{13}{3}$ to quantify more precisely the enthalpic stability of these configurations. This, however, is beyond the scope of the current study.

Figure 3 quantifies the structural correlations for water molecules in the vicinity of different topological defects in the H-bond network, by fixing the coordination state of only one of the two oxygen atoms involved. Of course, one could proceed to look into pair correlation functions for which both species are in a prescribed coordination state. This more detailed analysis reveals that in many cases there appear to be strong correlations between the position of defective coordination states. In other terms, when fluctuations in the generally tetrahedral network generate topological defects, such low-probability environments appear to be clustered close to each other. Henchman and coworkers have performed a similar analysis looking at the RDFs exclusively for water molecules that are different acceptor types. ${ }^{6}$ In particular, they observed for example, that water molecules that were single acceptors and triple acceptors tend to be close to each other. Here we considered more than 50 possible pairs of environments: we report all the results, for different models and simulation details, in the SI, and focus here on the most striking features that we could identify (Fig. 4).

Undercoordinated water molecules tend indeed to be strongly clustered together. The defectresolved RDF between $2{ }_{\mathrm{D}} 1_{\mathrm{A}}$ and $1_{\mathrm{D}} 2_{\mathrm{A}}$ environments shows a very pronounced peak at about $3.5 \AA$. Inspection of the directionally-resolved RDF reveals that this sharp peak is at least partly originating from the PAMM analysis that singles out a hydrogen bond in the act of breaking into a $2_{\mathrm{D}} 1_{\mathrm{A}}-1_{\mathrm{D}} 2_{\mathrm{A}}$ pair. However, the very broad angular spread of the peak at $3.5 \AA$ clearly paints a more complicated picture in which the weakening of the hydrogen bond is associated with greater conformational flexibility on both the donor and the acceptor side with respect to a tetrahedral $2{ }_{\mathrm{D}} 2_{\mathrm{A}}$ environment (see Fig. 3). In other terms, one can see this feature of hydrogen bonding as related to a form of entropic stabilization. In addition, $2{ }_{\mathrm{D}} 1_{\mathrm{A}}$ defects are associated with unusual angular correlations, with two very distinct peaks that can be seen at about $3.5 \AA$ distance, well separated from typical $\mathrm{H}$-bond directions. These are seen in the $2_{\mathrm{D}} 1_{\mathrm{A}} \rightarrow 1_{\mathrm{D}} 2_{\mathrm{A}}, 2_{\mathrm{D}} 2_{\mathrm{A}} \rightarrow 1_{\mathrm{D}} 2_{\mathrm{A}}$, and also in the $2{ }_{\mathrm{D}}{ }_{\mathrm{A}} \rightarrow 2_{\mathrm{D}} 1_{\mathrm{A}}$ correlations discussed in Ref. ${ }^{6}$ More generally, strong, anomalous 
Figure 4: Defect-resolved structural correlations in the hydrogen-bond network of vdW-corrected BLYP water. We focus on a few particularly significant pairs of under-coordinated and over-coordinated defects. As described in the main text, the structural correlations resemble some features seen in solid phases of ice. The crosses and circles correspond, respectively, to the positions of the nearest oxygen atoms in ice $\mathrm{Ih}$, and ice VIII expanded to match the density of room temperature water.

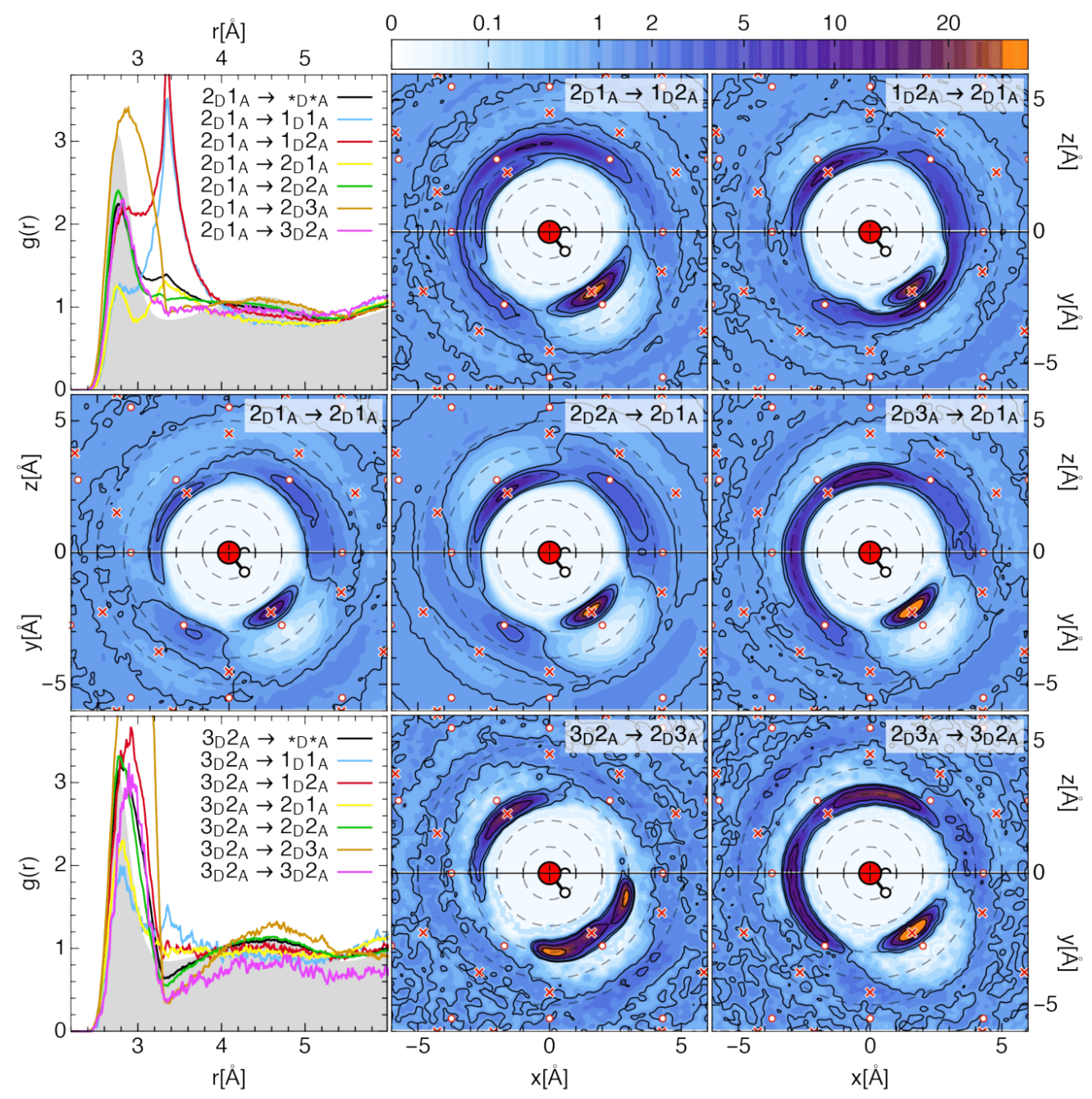

angular correlations are observed for all undercoordinated species (see also the whole series of defect-resolved 3D DFs in the SI), reinforcing the notion of the presence of a "weak" H-bonding mode that does not match the structural parameters range of a full-fledged hydrogen bond, but that contributes to the stability of coordination defects in liquid water.

In the liquid phase, the disorder and larger angular flexibility of water molecules makes it difficult to pinpoint the specific geometric features leading to the peculiar angular correlations. We thus turned to high density phases of water at lower temperature to understand the origins of these correlations. In Figure 4 we overlaid to the 3D O-
$\mathrm{O}$ distribution functions markers that indicate the position of the neighboring $\mathrm{O}$ atoms in ice $\mathrm{Ih}$ (crosses) and for a model of ice VIII expanded to match the density of room temperature water (circles). The standard hydrogen-bonded peaks correspond perfectly to the position of nearest neighbors in hexagonal ice, but the additional angular correlations found around undercoordinated waters are closely related to the coordination environments in ice VIII. A relationship between "interstitial" waters and high-density phases of ice was suggested in Ref. ${ }^{54}$ as a method to asses the accuracy of different electronic structure methods in describing defective environments in water. Our analysis confirms this intuition, and suggests that 
an even more representative benchmark could be obtained by expanding the unit cell to match the density of water at ambient conditions.

Fig. 4 also shows that $2{ }_{\mathrm{D}} 3_{\mathrm{A}}$ and $3{ }_{\mathrm{D}} 2_{\mathrm{A}}$ environments are very strongly correlated. In particular the $3{ }_{\mathrm{D}} 2_{\mathrm{A}} \rightarrow 2_{\mathrm{D}} 3_{\mathrm{A}}$ distribution function shows two distinct peaks at the brim of the donated H-bond region, suggesting that in most cases these configurations are associated with bifurcated $\mathrm{H}$-bonds. The inverse distribution $2{ }_{\mathrm{D}} 3_{\mathrm{A}} \rightarrow 3{ }_{\mathrm{D}} 2_{\mathrm{A}}$ shows the characteristic trigonal distribution of accepted $\mathrm{H}$ bonds, however with broader angular fluctuations that once again point at the increased flexibility associated with correlated defective environments.

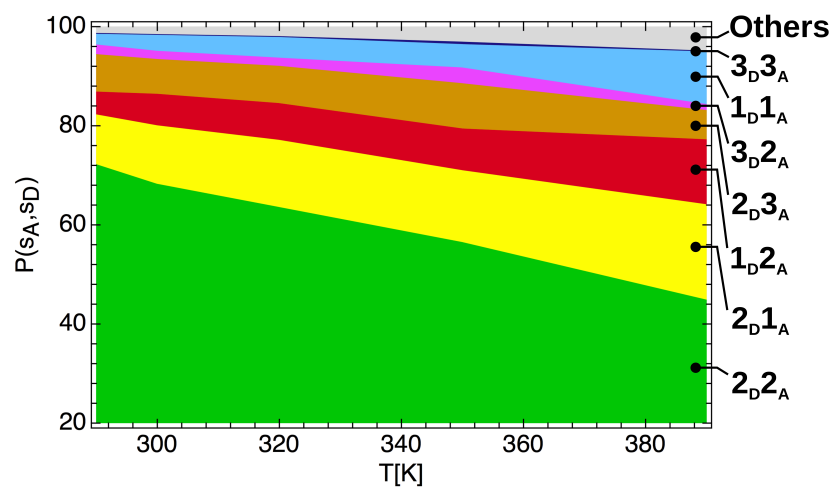

Figure 5: Temperature dependence (at constant room-temperature density) of the fraction of the main H-bonding defect states in the PTL simulations (BLYP+VDW, 128 water molecules).

\section{Comparison of Water Mod- els}

In the preceding discussions we have focused on the structural correlations of defects examining our production runs of the 128 water system at $300 \mathrm{~K}$ (PTL). In the following, we will now describe how defect populations and correlations change as a function of finite temperature, box size, the quality of the electronic structure and nuclear quantum effects. We find that topological defects appear to be present with similar concentrations across all the simulations. Furthermore, the structural correlations between them also appear to be qualitatively and sometimes even quantitatively conserved. However, the relative concentration of different defects changes in a subtle manner.

We begin by showing how the proportion of different types of structural defects change in water as a function of temperature at constant, roomtemperature density. As one moves from $290 \mathrm{~K}$ to $390 \mathrm{~K}$ (Fig. 5), there is a clear decrease, by about $25 \%$, in the proportion of water molecules that accept and donate two HBs. This is in turn accompanied by an increase in the number of structural defects. In particular, in going from $290 \mathrm{~K}$ to $390 \mathrm{~K}$ there is a significant increase in the number of water molecules accepting 2 and donating $1 \mathrm{HBs}$, accepting 1 and donating 2 hydrogen bonds and a smaller increase in the the number of defects accepting and donating 1 hydrogen bond. The concentration of overcoordinated defects such as the $2{ }_{\mathrm{D}}{ }_{\mathrm{A}}$ and $3{ }_{\mathrm{D}} 2_{\mathrm{A}}$ stays more or less constant, at least in the constant density conditions we are currently simulating. Despite the increase in the concentration of defects with growing temperature, the $3 \mathrm{D}$ correlation plots reveal the presence of well formed directed hydrogen bonds up to $390 \mathrm{~K}$ (see Figure. S5-S7 in the SI).

Fig. 6 shows the percentage of the main defects in the water network that we obtained with various simulation protocols. We compare the effect of finite box size (moving from 128 to 64 water molecules, that we used for most comparisons), the role of nuclear quantum effects and the use of model potentials such as TIP4P/2005 and the more recently developed MB-pol water model. For the most part, we see that the relative proportion of coordination defects is not very sensitive to the simulation protocol. In the case of MB-pol, the number of $2{ }_{\mathrm{D}} 2_{\mathrm{A}}$ waters is lower than that of BLYP+VDW PTWTE 64 by about 10\%, which is compensated by a slight increase in the under-coordinated $1_{\mathrm{D}} 2_{\mathrm{A}}$ and $2_{\mathrm{D}} 1_{\mathrm{A}}$ defects in the network. It is thus rather comforting to see that all these various protocols for simulating liquid water at ambient conditions produce qualitatively consistent results at least with respect to the types of coordination defects in the hydrogen bond network. On the other hand, the predictions for physical properties of water such as the RDF and the diffusion constant, do change significantly between these models. Seeing how such changes depend on small differences in the structure and stability 

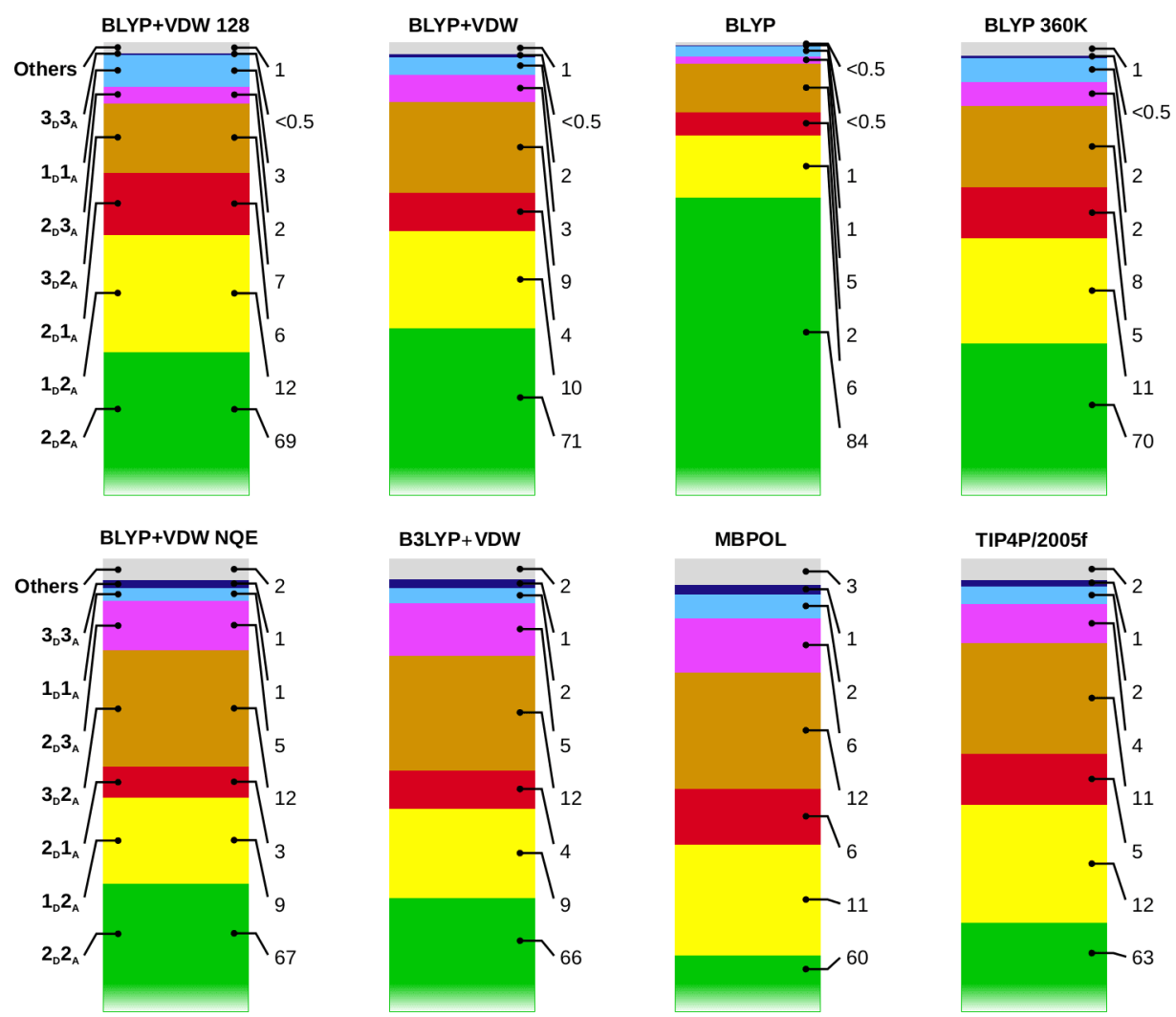

Figure 6: The bar-charts show the percentage of the main defect states considered in the text for different models explored in this work. The label on top of each chart indicates the simulation protocol (functional, system size, temperature, the inclusion of van-der-Waals corrections). On the extreme left, in bold, the defect type corresponding to each segment of the stack plots is indicated. The numbers on the right of each bar-chart indicate the percentage of each defect for the specified batch of simulations. Note that the segment corresponding to the majority, tetrahedral $22_{\mathrm{D}} 2_{\mathrm{A}}$ environments has been truncated for clarity.

of topological defects gives some rationale for the difficulty in obtaining a quantitatively accurate description of the structural and dynamical properties of water.

The clear outlier between the defect population plots in Fig. 6 is the case of the BLYP functional without $\mathrm{vdW}$ corrections that leads to a dramatic increase of the fully-coordinated tetrahedral environments. Indeed, it is well appreciated that standard generalized gradient approximation functionals used in AIMD simulations lead to the overstructuring of the hydrogen bonds in liquid water. A common trick that has been suggested in early CPMD simulations, and which has been used in many AIMD simulations thereafter, is to increase the temperature in the simulation which was applied to account for the supercooled nature of DFT water at $300 \mathrm{~K} .55$ Typical values that have been used are $330 \mathrm{~K}$ but there have also been suggestions that temperatures above $400 \mathrm{~K}$ are needed. ${ }^{[30}$
When this is done, it is empirically observed that the RDF gets less structured and agrees more closely with experiments. Indeed, we see that by raising the temperature to $360 \mathrm{~K}$ (the right-most stack plot in the first row of Fig. 6) one can reproduce quite accurately the defect populations obtained with vdW corrections.

Earlier, we alluded to the fact that one can think of the ensemble-averaged RDF as coming from individual contributions involving structural correlations between tetrahedral waters together with those from the clustering of different types of defects. To appreciate a bit better the challenge in converging this property, in Fig. 7 we compare the RDFs obtained with vdW corrections to those with the bare BLYP functional, at 300K, 360K and $390 \mathrm{~K}$, to illustrate how the choice of simulation temperature and the inclusion of dispersion interactions conspire to affect different parts of the distribution. It is clear that van-der-Waals interac- 


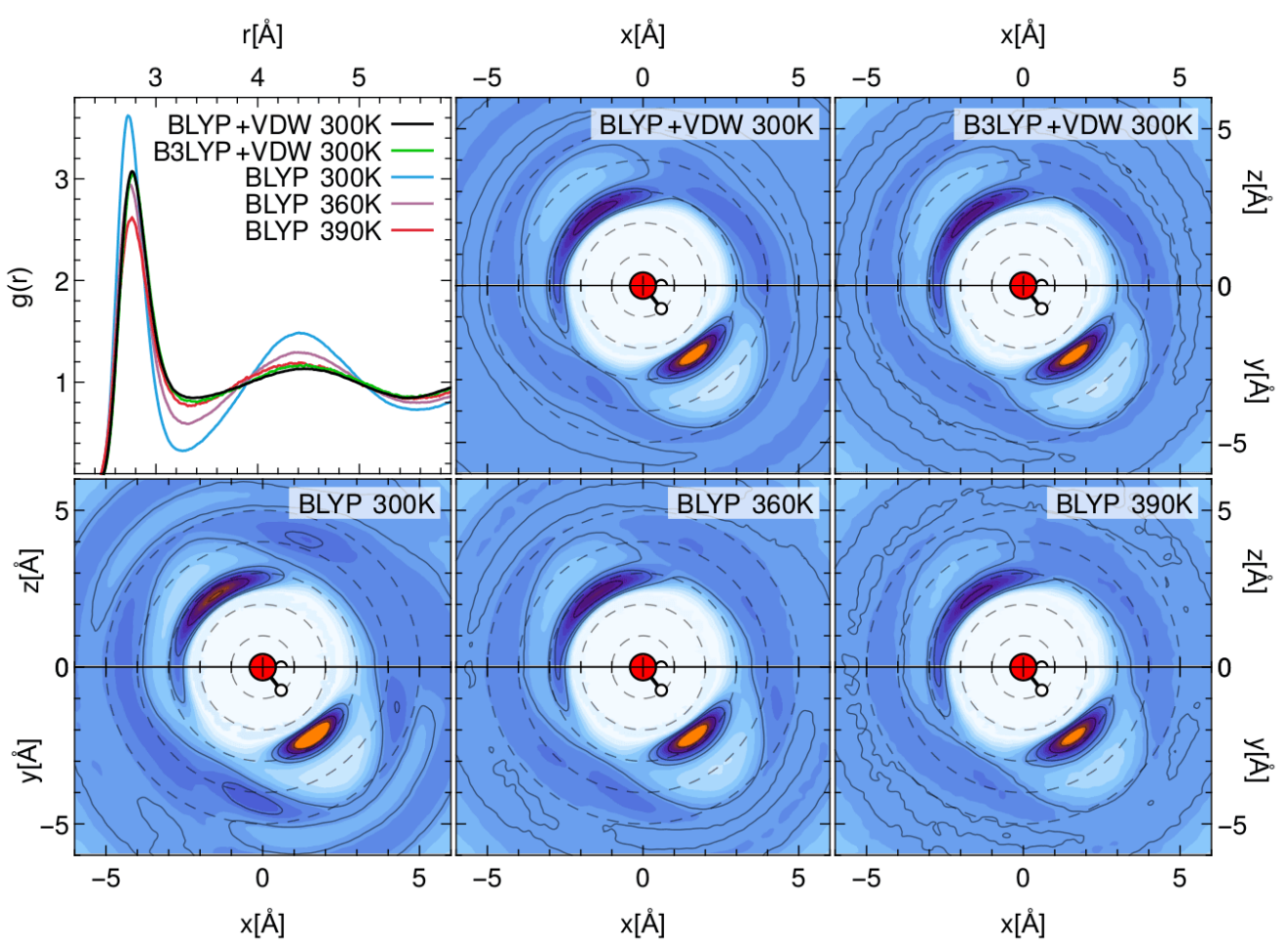

Figure 7: Radial and 3D O-O distribution functions, for selected simulation conditions. The bottom row compares BLYP-based simulations without vdW corrections at three different temperatures.

tions cannot be fully mimicked by an increase in simulation temperature. Without dispersion, the simulations at $360 \mathrm{~K}$ reproduce the height of the first peak of the distribution, but the long-range oscillations in the RDF are still considerably stronger than the vdW-corrected reference. One has to increase the temperature up to $390 \mathrm{~K}$ to approach the corrected long-range behavior, at the expense however of lowering too much the height of the first peak. Angular correlations, that are seen in the $3 \mathrm{D}$ distribution functions, clarify the source of this discrepancy. The bare GGA simulation shows pronounced peaks in the second coordination shell, that correspond to the angular positions seen in ice Ih (see also Fig 4). Raising the temperature broadens this peak, and shifts it towards the interstitial region that is characteristic of undercoordinated defects. At the same time, the increased temperature enhances the fluctuations and lowers the height of the first-neighbor peak. There is no temperature at which thermal fluctuations match the effect of vdW corrections on these two components simultaneously - performing simulations at an artificially increased temperature is a poor substitute for a model that describes properly dispersion interactions. vdW interactions thus play an important role in tuning both the short (first shell) and long-range (second shell and beyond parts of the RDF although the effect is more pronounced on the latter. Similar conclusions have also been made by Weeks and co-workers examining classical models of water such as SPC/E with molecular field theories. 56

There is currently an ongoing lively debate regarding the level of electronic structure theory required to reproduce structural and dynamical properties of liquid water. In particular, several studies have advocated for the need of including a certain amount of Hartree-Fock exchange in the exchange-correlation functional. ${ }^{27144}$ For this reason, we performed AIMD simulations with the B3LYP hybrid functional. Although we could not afford replica-exchange simulations, we ran four independent simulations at $300 \mathrm{~K}$, for a total of more than 60ps. The distributions with BLYP and B3LYP (both including dispersion interactions) show that the relative proportion of different types of coordination states are almost quantitatively the same. Also, radial and angular-resolved distribution functions are remarkably similar. Thus, while it has been previously observed that the use of hybrid functionals alone gives much better RDFs 
than bare GGAs,, 27 it appears that dispersion corrections can similarly remedy the most blatant deficiencies of BLYP, and that combining the two does not have a major effect compared to applying the exchange or vdW corrections separately.

The delicate balance between an ice-Ih-like and a defective hydrogen-bond network cannot be ascribed to a single approximation in the electronic structure framework. What is more, one should not focus too much on the RDF as the only benchmark to assess the quality of a water model: as we have shown, angular correlations and defectresolved distribution functions contain much more detailed information, and other physical properties (such as thermodynamic and dynamical properties,, 57 or isotope fractionation ratios 58 ) should also be included to avoid the risk of obtaining an RDF that matches experiment for the wrong reasons. Empirical vdW corrections seem to be enough to reproduce the experimental RDF, but there is strong evidence that this is largely due to a cancellation of errors between three and four-body terms,, 59 and it has been shown that the description of the water monomer energy is very poor in the absence of an exact exchange correction. $\underline{60}$

\section{Conclusions}

In this work we have used extensive AIMD simulations under a variety of different modelling conditions to understand the complex hydrogen bond landscape of liquid water. We have shown that due to topological constraints in the hydrogen bond network, water molecules that deviate from idealized tetrahedral structures cluster with each other with different propensities. In particular, we find that under-coordinated environments display consistently strong angular correlations that can be traced to a weaker, but still directional, mode of the hydrogen bond. This alternative H-bonding mode, that is not recognized as such by definitions that are trained to identify the majority tetrahedral environment, is related to the correlations found in a (dilated) ice VIII lattice. Although the focus of this work has been on structural correlations, these features have important bearing on understanding dynamical processes in liquid water. In particular, the clustering of these defects suggests that the breakage and formation of hydrogen bonds are correlated over an extended part of the network, and provides a mechanism to lower the barriers associated with hydrogen bond dynamics. In this regard, the type of analysis we have presented here forms a framework to rationalize, in a microscopic way, the balance between entropic and enthalpic forces that drive fluctuations in the hydrogen bond network.

Here, we took extra care to ensure that the AIMD simulations we used for analysis of the HB network were sampled extensively using ab initio replica exchange molecular dynamics. Furthermore, we examined the sensitivity of the topological properties of the hydrogen network to temperature, the use of dispersion corrections, the inclusion of exact exchange and nuclear quantum effects. Within the framework of ab initio methods, the inclusion of dispersion corrections appears to have the most significant impact on the RDF compared to the inclusion of exact exchange or nuclear quantum effects. It is rather comforting to see, however, that regardless of the details of the choice of the water potential, the qualitative predictions for the defect populations and structures are very similar. While there has been a lot of effort in trying to come up with simulation recipes to reproduce the experimental RDF, we find that the differences one observes from using different simulation protocols are quite subtle, and that for instance it is not possible to fully mimic the effect of dispersion corrections by altering the temperature of the simulation. Examining the sensitivity of defect distributions to the use of different simulation protocols, shows that there are noticeable differences in the relative populations of the various defects when varying minor computational details - for example, from the time step to the basis set (see SI). It should however be stressed that it is very hard to assess the statistical convergence of the minority populations, and that therefore we do not believe one can draw meaningful conclusions on the significance of these differences.

It is clear that the radial distribution function masks a lot of complexity in the underlying hydrogen bond network and that the details of its structure will be modulated by the balance in the relative proportions of different defects and how they cluster with each other - two aspects that can 
be disentangled by looking at three dimensional distribution functions resolved in different defect pairs. We believe that the analysis framework we introduce here, based on a self-consistent, datadriven definition of the hydrogen bond and the study of non-trivial correlations between topological defects, will prove to be particularly effective when investigating different portions of the phase diagram of bulk water, the role of charged defects as well as the behaviour of water in confinement and at interfaces.

\section{Acknowledgements}

We would like to acknowledge generous allocation of CPU time by CSCS under the project id s466 and s553. MC and PG would like to acknowledge funding from CCMX and from the MPG-EPFL center for molecular nanoscience.

See the supporting information for the details of all of the simulations referenced in this work, a discussion of the impact of the H-bond definition, and the complete set of defect population and correlation analysis. This information is available free of charge via the Internet at http://pubs.acs.org

\section{References}

(1) Bergman, D. L. Chem. Phys. 2000, 253, 267 -282 .

(2) Agmon, N. Acc. Chem. Res. 2012, 45, 63-73.

(3) Kühne, T. D.; Khaliullin, R. Z. Nat. Commun. 2013, 4, 1450.

(4) Ceriotti, M.; Cuny, J.; Parrinello, M.; Manolopoulos, D. E. Proc. Nat. Acad. Sci. U.S.A. 2013, 110, 15591-15596.

(5) Sciortino, F.; Fornili, S. L. J. Chem. Phys. 1989, 90, 2786-2792.

(6) Henchman, R. H.; Irudayam, S. J. J. Phys. Chem. B 2010, 114, 16792-16810.

(7) Kirchner, B.; di Dio, P. J.; Hutter, J. Multiscale Molecular Methods in Applied Chemistry; Topics in Current Chemistry; SpringerVerlag Berlin, 2012; Vol. 307; pp 109-153.
(8) Sprik, M.; Hutter, J.; Parrinello, M. J. Chem. Phys. 1996, 105, 1142-1152.

(9) Todorova, T.; Seitsonen, A. P.; Hutter, J.; Kuo, I.-F. W.; Mundy, C. J. J. Phys. Chem. B 2006, 110, 3685-3691.

(10) Guidon, M.; Schiffmann, F.; Hutter, J.; VandeVondele, J. J. Chem. Phys. 2008, 128, 214104.

(11) Silvestrelli, P. L.; Parrinello, M. Phys. Rev. Lett. 1999, 82, 3308-3311.

(12) Grossman, J. C.; Schwegler, E.; Draeger, E. W.; Gygi, F.; Galli, G. J. Chem. Phys. 2004, 120, 300-311.

(13) Fernandez-Serra, M. V.; Artacho, E. J. Chem. Phys. 2004, 121, 11136-11144.

(14) Sit, P. H.-L.; Marzari, N. J. Chem. Phys. 2005, 122, 204510.

(15) VandeVondele, J.; Mohamed, F.; Krack, M.; Hutter, J.; Sprik, M.; Parrinello, M. J. Chem. Phys. 2005, 122, 014515.

(16) Lee, H.-S.; Tuckerman, M. E. J. Chem. Phys. 2006, 125, 154507.

(17) Kuo, I.-F. W.; Mundy, C. J.; McGrath, M. J.; Siepmann, J. I.; VandeVondele, J.; Sprik, M.; Hutter, J.; Chen, B.; Klein, M. L.; Mohamed, F.; Krack, M.; Parrinello, M. J. Phys. Chem. B 2004, 108, 12990-12998.

(18) Zhang, C.; Donadio, D.; Gygi, F.; Galli, G. J. Chem. Theo. Comput. 2011, 7, 1443-1449.

(19) Lin, I.-C.; Seitsonen, A. P.; CoutinhoNeto, M. D.; Tavernelli, I.; Rothlisberger, U. J. Phys. Chem. B 2009, 113, 1127-1131.

(20) Santra, B.; Michaelides, A.; Scheffler, M. J. Chem. Phys. 2009, 131.

(21) Jonchiere, R.; Seitsonen, A. P.; Ferlat, G.; Saitta, A. M.; Vuilleumier, R. J. Chem. Phys. 2011, 135, 154503.

(22) Zhang, C.; Wu, J.; Galli, G.; Gygi, F. J. Chem. Theo. Comput. 2011, 7, 3054-3061. 
(23) Møgelhøj, A.; Kelkkanen, A. K.; Wikfeldt, K. T.; Schiøtz, J.; Mortensen, J. J.; Pettersson, L. G. M.; Lundqvist, B. I.; Jacobsen, K. W.; Nilsson, A.; Nørskov, J. K. J. Phys. Chem. B 2011, 115, 14149-14160.

(24) Chen, B.; Ivanov, I.; Klein, M. L.; Parrinello, M. Phys. Rev. Lett. 2003, 91, 215503.

(25) Morrone, J. A.; Car, R. Phys. Rev. Lett. 2008, $101,017801$.

(26) Del Ben, M.; Schönherr, M.; Hutter, J.; Vandevondele, J. J. Phys. Chem. Lett. 2013, 4, 3753-3759.

(27) DiStasio, R. A.; Santra, B.; Li, Z.; Wu, X.; Car, R. J. Chem. Phys. 2014, 141,--

(28) Bonomi, M.; Parrinello, M. Phys. Rev. Lett. 2010, 104, 190601.

(29) Gasparotto, P.; Ceriotti, M. J. Chem. Phys. 2014, 141, 174110.

(30) Yoo, S.; Xantheas, S. S. J. Chem. Phys. 2011, $134,-$.

(31) Akin-Ojo, O.; Wang, F. Chem. Phys. Lett. 2011, 513, $59-62$.

(32) Stanley, H. E.; Teixeira, J. J. Chem. Phys. 1980, 73, 3404-3422.

(33) Soper, A. K.; Phillips, M. G. Chem. Phys. 1986, 107, 47-60.

(34) Hassanali, A.; Giberti, F.; Cuny, J.; Kühne, T. D.; Parrinello, M. Proc. Nat. Acad. Sci. U.S.A. 2013, 13723.

(35) VandeVondele, J.; Krack, M.; Mohamed, F.; Parrinello, M.; Chassaing, T.; Hutter, J. Comput. Phys. Commun. 2005, 167, 103-128.

(36) Ceriotti, M.; More, J.; Manolopoulos, D. E. Comput. Phys. Commun. 2014, 185, 10191026.

(37) Grimme, S.; Antony, J.; Ehrlich, S.; Krieg, H. J. Chem. Phys. 2010, 132, 154104.

(38) Lee, C.; Yang, W.; Parr, R. G. Phys. Rev. 1988, B37, 785-789.
(39) Goedecker, S.; Teter, M.; Hutter, J. Phys. Rev. 1996, B54, 1703-1710.

(40) Bussi, G.; Donadio, D.; Parrinello, M. J. Chem. Phys. 2007, 126, 14101.

(41) Ceriotti, M.; Bussi, G.; Parrinello, M. Phys. Rev. Lett. 2009, 102, 020601.

(42) Ceriotti, M.; Bussi, G.; Parrinello, M. J. Chem. Theo. Comput. 2010, 6, 1170-1180.

(43) Ceriotti, M.; Manolopoulos, D. E. Phys. Rev. Lett. 2012, 109, 100604.

(44) Guidon, M.; Hutter, J.; VandeVondele, J. J. Chem. Theo. Comput. 2010, 6, 2348-2364.

(45) González, M. a.; Abascal, J. L. F. J. Chem. Phys. 2011, 135, 224516.

(46) Babin, V.; Leforestier, C.; Paesani, F. J. Chem. Theo. Comput. 2013, 9, 5395-5403.

(47) Babin, V.; Medders, G. R.; Paesani, F. J. Chem. Theo. Comput. 2014, 10, 1599-1607.

(48) Medders, G. R.; Babin, V.; Paesani, F. J. Chem. Theo. Comput. 2014, 10, 2906-2910.

(49) Kumar, R.; Schmidt, J. R.; Skinner, J. L. J. Chem. Phys. 2007, 126, 204107.

(50) Laage, D.; Hynes, J. T. J. Phys. Chem. B 2008, 112, 14230-14242.

(51) Lawrence, C.; Skinner, J. Chem. Phys. Lett. 2003, 369, 472-477.

(52) Auer, B.; Kumar, R.; Schmidt, J. R.; Skinner, J. L. Proc. Natl. Acad. Sci. USA 2007, 104, 14215-14220.

(53) Wikfeldt, K. T.; Nilsson, A.; Pettersson, L. G. M. Phys. Chem. Chem. Phys. 2011, 13, 19918-19924.

(54) Gillan, M. J.; Alfè, D.; Bartók, a. P.; Csányi, G. J. Chem. Phys. 2013, 139, 244504.

(55) Chen, W.; Sharma, M.; Resta, R.; Galli, G.; Car, R. Phys. Rev. B: Condens. Matter Mater. Phys. 2008, 77, 245114. 
(56) Remsing, R. C.; Rodgers, J. M.; Weeks, J. D. Journal of Statistical Physics 2011, 145, 313-334.

(57) Kühne, T. D.; Krack, M.; Parrinello, M. J. Chem. Theo. Comput. 2009, 5, 235-241.

(58) Wang, L.; Ceriotti, M.; Markland, T. E. J. Chem. Phys. 2014, 141, 104502.

(59) Medders, G. R.; Götz, A. W.; Morales, M. A.; Bajaj, P.; Paesani, F. $J$. Chem. Phys. 2015, 143, 104102.

(60) Morales, M. a.; Gergely, J. R.; McMinis, J.; McMahon, J. M.; Kim, J.; Ceperley, D. M. J. Chem. Theo. Comput. 2014, 10, 2355-2362. 